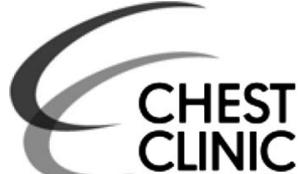
Dr Bernie Young Sunwoo, Division of Pulmonary, Critical Care and Sleep Medicine, Department of Medicine, University of California San Francisco, 2330 Post St, Suite 420, San Francisco, CA 94115 USA; bernie.sunwoo@ucsf.edu

Received 17 June 2016 Revised 14 October 2016 Accepted 29 November 2016 Published Online First 23 December 2016

CrossMark

\title{
An oesophageal and pulmonary association not to forget
}

\author{
Bernie Young Sunwoo
}

A 45 -year-old Indian man with a history of achalasia presented with fevers, cough and fatigue. He had undergone endoscopic dilatation for achalasia several years prior. Chest radiograph revealed a dilated oesophagus and a left perihilar opacity (figure 1). He was treated for community-acquired pneumonia with a course of clarithromycin but had persistent cough, fatigue and weight loss with nonresolving radiographic infiltrates. A 3-week course of amoxicillin clavulanate was prescribed for suspected aspiration with little clinical improvement, and a chest CT was performed (figure 2). The patient had never smoked and had moved to the USA from India over 20 years ago, last travelling to India 1 year prior. Prior purified protein derivative skin tests (PPDs) were negative. HIV testing was negative.

Induced sputums isolated Mycobacterium fortuitum. Bronchoscopy was performed showing no endobronchial lesions. Bronchoalveolar lavage from the left lower lobe also grew $M$. fortuitum. Mycobacterium tuberculosis PCR and all other cultures were negative. Cytology was negative.

M. fortuitum is a rapidly growing nontuberculous mycobacterium that is ubiquitous in the environment. It has been associated with infections of the skin, soft tissue, wounds and bone. Pulmonary infection is uncommon in the immunocompetent host with no underlying lung disease except in the setting of gastro-oesophageal

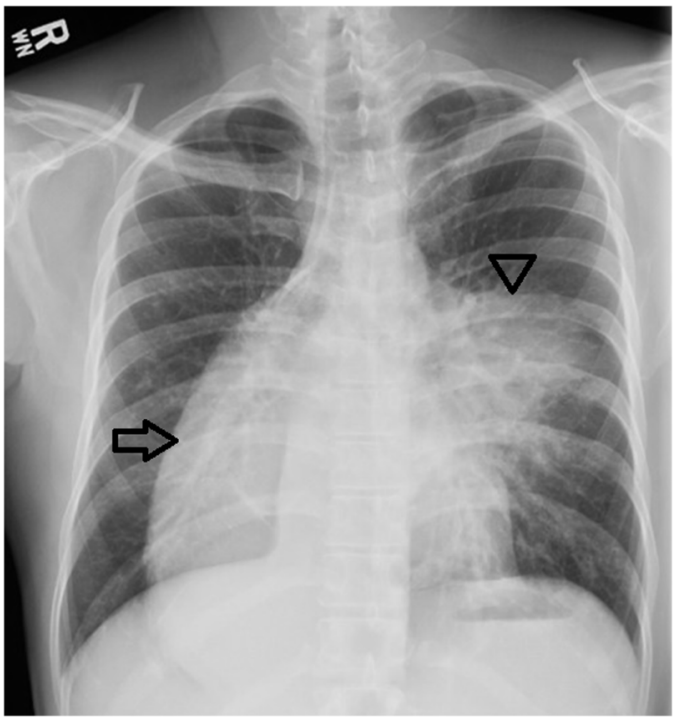

Figure 1 Chest radiography showing a dilated oesophagus (arrow) and left perihilar opacification (arrowhead). disease. $^{1-4}$ An association between achalasia and M. fortuitum pulmonary infection has been described. Lipoid ingestion and physical protection of the mycobacteria by surrounding fat has been proposed as one mechanism supporting survival against enzymes and growth of the mycobacteria. $^{2} 56$ The stagnant oesophageal contents in achalasia have also been proposed to support mycobacterial growth, such that regurgitation and aspiration of large quantities of mycobacteria into the lungs may result in infection. ${ }^{2} 56$

In a retrospective review of 182 patients with positive respiratory specimen for $M$. fortuitum, Park et $\mathrm{al}^{7}$ suggested that M. fortuitum represented colonisation or transient infection but no data on oesophageal disease were provided. Given the described association between achalasia and M. fortuitum, our patient was initiated on linezolid,
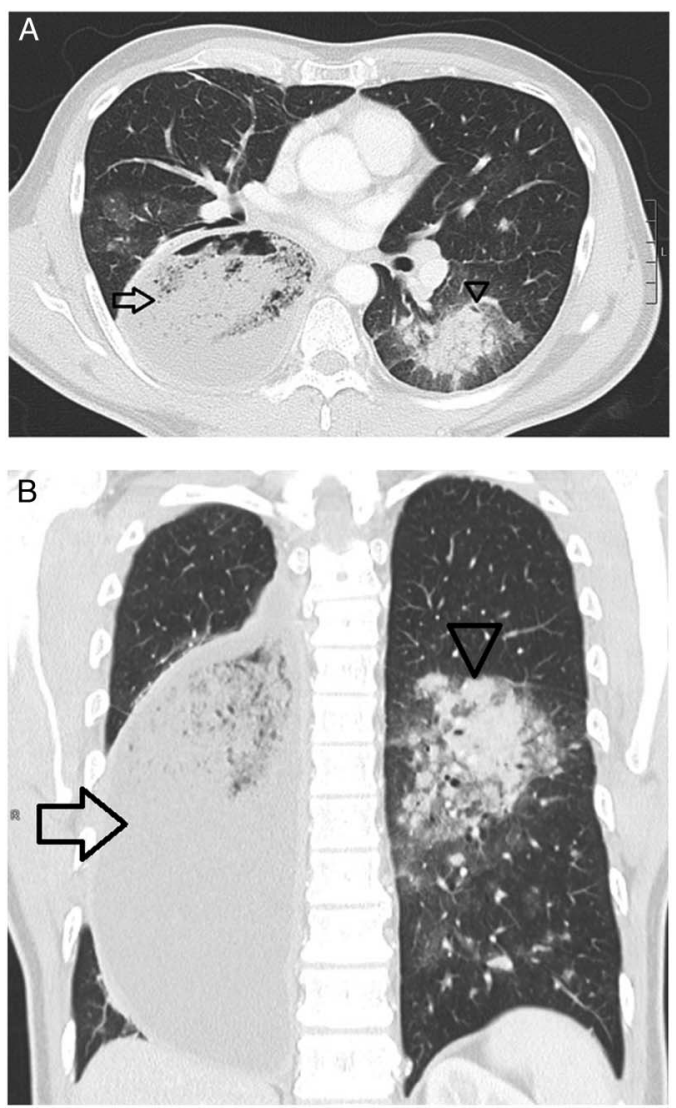

Figure 2 Chest CT. (A) Axial view and (B) coronal view showing a markedly dilated oesophagus with fluid and debris (arrow) with gradual tapering at the gastrooesophageal junction and patchy consolidation in the left lower lobe (arrowhead). 


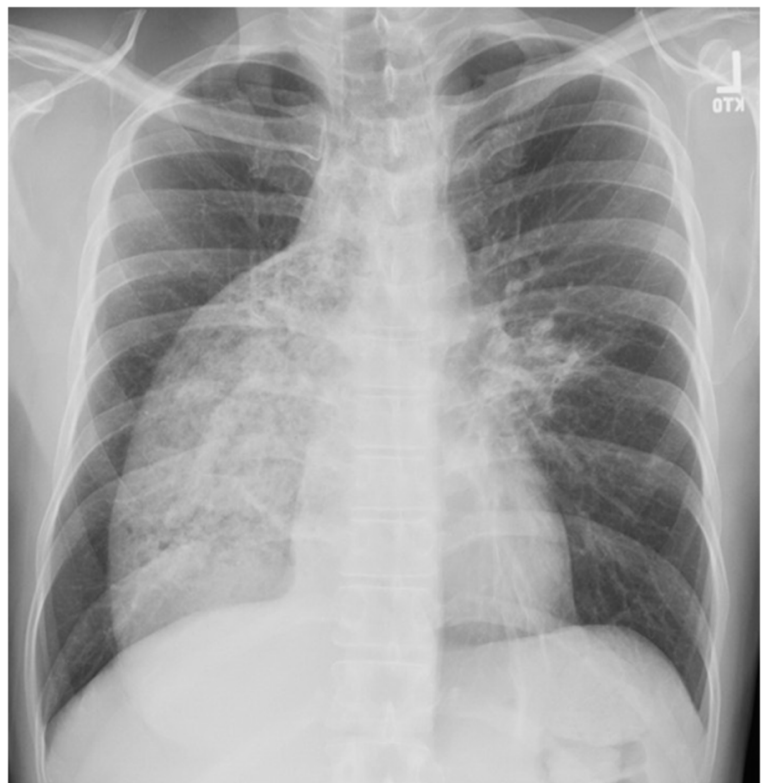

Figure 3 Repeat chest radiography after 1 month of Mycobacterium Fortuitum antimicrobial therapy showing improvement in the left perihilar opacity.

moxifloxacin and co-trimoxazole based on susceptibility testing, with clinical and radiographic improvement 1 month later (figure 3). The optimal antimicrobial therapy for pulmonary $M$. fortuitum remains unknown and is guided by antibiotic susceptibilities. Treatment with at least two agents with in vitro susceptibility has been recommended for at least 12 months of negative sputum cultures. ${ }^{1} \mathrm{M}$. fortuitum isolates are often susceptible to multiple antimicrobial agents including the quinolones, newer macrolides, doxycycline, minocycline and sulfonamides but an inducible erythromycin methylase erm gene that confers resistance to the macrolides has been shown. ${ }^{1}$ Accordingly, cautious use of macrolides has been suggested. ${ }^{1}$ After 4 weeks, our patient discontinued linezolid due to intolerance and he remained on moxifloxacin and co-trimoxazole with plans to complete 12 months of therapy from the time of his first negative sputum culture. Gastroenterology and surgical consultations were obtained to optimise the management of his oesophageal disease and minimise the risk of aspiration. In conclusion, M. fortuitum is an uncommon pulmonary infection, but in patients with achalasia, the association is one not to forget.

Competing interests None declared.

Patient consent Obtained.

Provenance and peer review Not commissioned; externally peer reviewed.

\section{REFERENCES}

1 Griffith DE, Aksamit T, Brown-Elliott BA, et al. An official ATS/IDSA statement: diagnosis, treatment, and prevention of nontuberculous mycobacterial diseases. Am J Respir Crit Care Med 2007:175:367-416.

2 Aronchick JM, Miller WT, Epstein DM, et al. Association of achalasia and pulmonary Mycobacterium fortuitum infection. Radiology 1986;160:85-6.

3 Banerjee R, Hall R, Hughes GR. Pulmonary Mycobacterium fortuitum infection in association with achalasia of the oesophagus. Case report and review of the literature. Br J Dis Chest 1970:64:112-18.

4 Griffith DE, Girard WM, Wallace RJ Jr. Clinical features of pulmonary disease caused by rapidly growing mycobacteria. An analysis of 154 patients. Am Rev Respir Dis 1993;147:1271-8.

5 Hadjiliadis D, Adlakha A, Prakash UB. Rapidly growing mycobacterial lung infection in association with esophageal disorders. Mayo Clin Proc 1999;74:45-51.

6 Varghese $G$, Shepherd $R$, Watt $P$, et al. Fatal infection with Mycobacterium fortuitum associated with oesophageal achalasia. Thorax 1988;43:151-2.

7 Park S, Suh GY, Chung MP, et al. Clinical significance of Mycobacterium fortuitum isolated from respiratory specimens. Respir Med 2008;102:437-42. 